\title{
Percepção acadêmica sobre metodologias ativas: um relato de experiência sobre a criação de videoaulas por meio da Aprendizagem Baseada em Projetos
}

\author{
Academic perception on active methodologies: an experience report on creating video classes \\ through Project-Based Learning \\ Percepción académica sobre metodologías activas: informe de experiencia sobre la creación de \\ video clases a través del Aprendizaje Basado en Proyectos
}

Recebido: 18/09/2021 | Revisado: 25/09/2021 | Aceito: 26/09/2021 | Publicado: 27/09/2021

\author{
Brenda Beatriz Silva Monteiro \\ ORCID: https://orcid.org/0000-0002-2682-3051 \\ Universidade do Estado do Pará, Brasil \\ E-mail: bbeatrizfisio@gmail.com \\ Elissa Raissa Siqueira do Nascimento \\ ORCID: https://orcid.org/0000-0002-7819-981X \\ Universidade do Estado do Pará, Brasil \\ E-mail: elissaraissa@gmail.com \\ Daniela Ferreira Leite \\ ORCID: https://orcid.org/0000-0001-9926-9226 \\ Universidade do Estado do Pará, Brasil \\ E-mail: danielafleite1@gmail.com \\ Erica Silva de Souza Matsumura \\ ORCID: https://orcid.org/0000-0003-4844-8050 \\ Universidade do Estado do Pará, Brasil \\ E-mail: erica.s.souza@terra.com.br
}

\begin{abstract}
Resumo
Objetivo: compreender a percepção acadêmica sobre metodologias ativas por meio da experiência sobre a criação de videoaulas através da Aprendizagem Baseada em Projetos. Metodologia: relato de experiência, observacional, de caráter qualitativo desenvolvido em um debate com alunos de uma Universidade em Belém/PA. A vivência foi efetivada pelos acadêmicos do Curso de Fisioterapia, durante a disciplina de Biofísica, no mês de maio de 2018. Foi realizado um encontro com alunos e professores da Instituição no momento da administração da aula, enfatizando a Aprendizagem Baseada em Projetos e as dificuldades da metodologia tradicional. Resultados: o principal aspecto destacado na vivência foi a percepção dos acadêmicos sobre metodologia ativa por meio de vídeoaula através dos conceitos de Excelente, Bom, Regular, Ruim ou Péssimo. O conceito Excelente foi mais eleito pelos alunos, com mais de $2 / 3$ do total dos estudantes. Conclusão: a vivência permitiu uma melhor compreensão da percepção dos acadêmicos sobre as metodologias ativas, sobretudo a Aprendizagem Baseada em Projetos por meio de vídeoaulas. Sendo assim, é imprescindível mais estudos sobre influência da Aprendizagem Baseada em Projetos no aprendizado dos alunos.
\end{abstract}

Palavras-chave: Aprendizagem baseada em projetos; Metodologia ativa; Aprendizagem; Fisioterapia; Ensino.

\begin{abstract}
Objective: to understand the academic perception of active methodologies through the experience of creating video classes through Project Based Learning. Methodology: qualitative, observational, experience report developed in a debate with students from a University in Belém / PA. The experience was carried out by the students of the Physiotherapy Course, during the course of Biophysics, in the month of May 2018. A meeting was held with students and teachers of the Institution at the time of class administration, emphasizing Project-Based Learning and the difficulties traditional methodology. Results: the main aspect highlighted in the experience was the students' perception of active methodology through video lessons through the concepts of Excellent, Good, Regular, Bad or Very Bad. The Excellent concept was most chosen by the students, with more than $2 / 3$ of the total students. Conclusion: the experience allowed a better understanding of the students' perception of active methodologies, especially Project-Based Learning through video lessons. Therefore, further studies on the influence of Project Based Learning on student learning are essential.
\end{abstract}

Keywords: Project-based learning; Active methodology; Learning; Physiotherapy; Teaching.

\section{Resumen}

Objetivo: comprender la percepción académica de las metodologías activas a través de la experiencia de crear video clases a través del Aprendizaje Basado en Proyectos. Metodología: relato de experiencia cualitativo, observacional 
desarrollado en un debate con estudiantes de una Universidad de Belém / PA. La experiencia fue realizada por los alumnos del Curso de Fisioterapia, durante el curso de Biofísica, en mayo de 2018. Se realizó un encuentro con alumnos y docentes de la Institución en el momento de la administración de la clase, enfatizando el Aprendizaje Basado en Proyectos y las dificultades tradicionales. metodología. Resultados: el aspecto principal destacado en la experiencia fue la percepción de los estudiantes de la metodología activa a través de lecciones en video a través de los conceptos de Excelente, Bueno, Regular, Malo o Muy Malo. El concepto de Excelente fue más elegido por los estudiantes, con más de $2 / 3$ del total de estudiantes. Conclusión: la experiencia permitió una mejor comprensión de la percepción de los estudiantes sobre las metodologías activas, especialmente el Aprendizaje Basado en Proyectos a través de lecciones en video. Por lo tanto, es esencial realizar más estudios sobre la influencia del aprendizaje basado en proyectos en el aprendizaje de los estudiantes.

Palabras clave: Aprendizaje basado en proyectos; Metodología activa; Aprendiendo; Fisioterapia; Enseñanza.

\section{Introdução}

Nos últimos anos, a educação vem admitindo mudanças, principalmente em relação à estrutura curricular tradicional, a qual considera o assunto repassado como principal ferramenta. Esta prática pedagógica se baseia na concepção que a educação é o ato de depositar, transferir e reproduzir valores e conhecimentos (Moran, 2015; Ribeiro, et al., 2016; Brasil, 2018; Alves, et. al., 2021).

Assim, as tendências atuais da educação orientam para a utilização de metodologias ativas, de modo a fornecer protagonismo aos estudantes e ao processo de aprendizagem, responsabilizando-os, em parte, por sua própria formação (Moraes \& Paim, 2017). Para tanto, deve-se estimular uma visão holística, incentivando a construção de redes sociais (dentro e fora do espaço universitário) que possibilitem a expansão da consciência individual e coletiva. Diante disso, percebe-se que as mudanças na aprendizagem na atual sociedade trazem a necessidade de um novo perfil docente (Diesel; Baldez \& Martins, 2017; Moraes, et al., 2017; Cecílio \& Reis, 2018).

Com isso, a evolução na formação de professores é uma necessidade, possuindo como pressuposto a diversidade de conhecimentos à sua prática profissional, com base em posturas reflexivas, investigativas e críticas (Schmitz \& Reis, 2018). Neste sentido, as metodologias ativas surgem para referir-se a uma variedade de estratégias de ensino, como: Aprendizagem Baseada em Problemas (ABP), problematização, Aprendizagem Baseada em Projetos (ABP), método do caso e sala de aula invertida dentre outras (Sobral \& Campos, 2012; Farias; Ribeiro; Anjos \& Brito, 2018).

Essas estratégias são idealizadas a partir de métodos de ensino fundamentadas na concepção pedagógica críticoreflexivo, por meio de uma atuação em contextos de vida real, intervindo sobre a realidade, de forma a estimular a interação entre os diversos atores. Desse modo, incentiva-se a valorização da construção coletiva do conhecimento em seus diferentes saberes e cenários de aprendizagem (Lima, 2017; Grillo; Ibarra \& Mezadri, 2019).

A ABP tem sido utilizada no ensino como método para aprendizagem, utilizando tarefas realistas baseadas em problemas. Além disso, requer iniciativa e independência dos alunos, resultando na produção de um produto final. Os projetos envolvem estudantes que trabalhem em grupos para promover a colaboração e desenvolver competências interpessoais (Emery \& Morgan, 2017; Rios; Sousa \& Caputo, 2019).

A metodologia ativa ABP, também pode ser potencializada pelas tecnologias digitais, na medida que os passos da resolução do problema podem apresentar-se de várias possibilidades à aprendizagem por projetos. Podendo ser utilizado diferentes fontes de informação e estratégias de aprendizado, inclusive locais diferentes da sala de aula, materiais e equipamentos diversos a dependendo da natureza do projeto (Borges \& Alencar, 2017; Vendruscolo; Trindade; Maffissoni; Martini; Silva Filho \& Sandri, 2020).

Como consequência, essas práticas devem estimular a criatividade na construção de soluções aos problemas da vida real, promovendo assim, a liberdade de pensar e agir (Borges \& Alencar, 2017). Porém, para a consolidação das metodologias 
ativas, é necessária a reorganização da matriz curricular dos cursos, com capacitação contínua dos professores e estruturação das Universidades para oferecer o campo adequado de desenvolvimento intelectual proposto ao acadêmico.

Sendo assim, presente estudo teve como objetivo compreender a percepção acadêmica sobre metodologias ativas por meio da experiência sobre a criação de videoaulas através da ABP no curso de Fisioterapia de uma Instituição de Ensino Superior privada, na disciplina de Biofísica.

\section{Metodologia}

Trata-se de um relato de experiência do tipo descritivo, observacional de caráter qualitativo, é uma pesquisa de campo, desenvolvido em uma Instituição de Ensino Superior (IES) em Belém-PA-Brasil. A referida vivência foi realizada durante a disciplina de Biofísica em maio de 2018. Além disso, a pesquisa também teve como suporte metodológico feito por Ludke, M. e Andre, M. E . D. A. (2013). Pesquisas em educação: uma abordagem qualitativa. São Paulo: E.P.U.

A Coordenação Pedagógica da Instituição, em consonância com os Professores responsáveis pela devida disciplina, analisou o histórico das notas dos alunos e a forma como era administrada o conteúdo; por conta disso, reuniram-se em um debate.

No primeiro momento, foi realizado pelos discentes e docentes uma roda de conversa a fim de debater sobre a metodologia de ensino ABP. Entre as discussões que mais chamou atenção, foi que a disciplina de Biofísica era administrada pelo método tradicional de ensino, o que tornava o conteúdo exaustivo e extenso, ocasionando baixa adesão às aulas. Por isso, a IES decidiu, em conjunto aos professores, utilizar a ABP, nesta disciplina, a fim de melhorar o aceite e aderência dos alunos às aulas do módulo de Biofísica.

Em seguida, os professores informaram a respeito da $\mathrm{ABP}$ e orientaram a formarem grupos de quatro, sendo divididos aleatoriamente com os respectivos sistemas do corpo humano: visão, audição, olfato e respiração. A turma era formada por 18 alunos, de ambos os gêneros, de idades variadas, sendo divididos em: 4 para visão e audição (cada) e 5 para olfato e respiração (cada).

Assim, depois de formados os grupos, foi decidido que cada um produziria uma vídeoaula a respeito de cada tema, podendo utilizar os laboratórios de anatomia e recursos áudiovisuais (tabletes, computadores, celulares, filmadoras). Além disso, foi possível acompanhar a construção dos projetos durante quatro semanas; sendo, assim, finalizados.

Foi observado algumas dúvidas dos alunos referentes a atividade proposta, pois não estavam habituados a nova metodologia. Sendo assim, foi proposto, pelos professores, que cada grupo fosse orientado pelo docente da disciplina, durante as quatro semanas consecutivas, no momento da aula de Biofísica.

Depois disso, percebeu-se que os alunos começaram a elaborar, planejar e executar, de forma mais proativa, os conteúdos de vídeoaula, utilizando diversas estratégias, como: apresentando um telejornal, realizando entrevista ou executando uma experiência no laboratório de anatomia, com a utilização de recursos áudiovisuais.

Finalizado o período para a construção das vídeoaulas, houve o encontro para apresentação dos produtos, sanando algumas dúvidas acerca do conteúdo apresentado.

Sendo assim, a partir dessas vivências, gerou-se questionamentos se de fato os alunos teriam aprendido os conteúdos de Biofísica com base na nova metodologia ABP, por meio das vídeoaulas. Diante disso, os questionamentos foram realizados, com cada aluno, dando conceitos de Excelente, Bom, Regular, Ruim ou Péssimo. Assim, objetivando compreender a percepção acadêmica sobre metodologias ativas, nesse caso, a ABP.

Outrossim, é imprescindível frisar que na ABP o professor também é fundamental para que a metodologia de ensino seja eficaz; assim como foi importante a sua participação nas seguintes experiências: 
$\checkmark$ Acompanhar na montagem dos projetos a fim de sanar dúvidas e orientar melhor os alunos quanto a construção das vídeoaulas;

$\checkmark$ Debater artigos ou plataformas online de materiais em que os alunos pudessem estudar sobre o conteúdo;

$\checkmark$ Estimular os alunos quanto a criatividade de repassar o projeto final com auxílio de aulas de Tecnologia de Informação para melhor manuseio das ferramentas de informática;

$\checkmark$ Organizar as vídeoaulas, em sala de aula, com os alunos na divisão dos conteúdos da disciplina.

\section{Resultados e Discussão}

Os dados qualitativos foram utilizados em convergência com as informações de pesquisas de outras literaturas. O principal aspecto destacado foi a percepção de acadêmicos sobre metodologia ativa por meio de vídeoaula por meio dos conceitos de Excelente, Bom, Regular, Ruim ou Péssimo.

Tabela 1. Percepção acadêmica sobre metodologia ativa por meio de vídeoaula.

\begin{tabular}{c|c|c|c|c} 
CONCEITO & E (Excelente) & B (Bom) & R (Regular) & $\begin{array}{c}\text { R (Ruim ou } \\
\text { Péssimo) }\end{array}$ \\
\hline $\begin{array}{c}\text { AMOSTRA E } \\
\text { PORCENTAGEM }\end{array}$ & $8(44,4 \%)$ & $5(27,7 \%)$ & $4(22,2 \%)$ & $1(5,55 \%)$
\end{tabular}

Fonte: Autores (2018).

Os achados mostram 8(44,4\%) dos alunos deram o conceito Excelente; Bom 5(27,7\%), Regular 4(22,2\%) e Ruim ou Péssimo $1(5,55 \%)$.

Além disso, maioria dos discentes atribuiu conceito Excelente ou Bom à metodologia $(72,1 \%)$, enquanto que apenas 1 voluntário considerou Péssimo. Esse fato mostra que os alunos acreditaram que o método proposto é válido, gerando uma importante discussão sobre o novo modelo de ensino.

Com isso, percebeu-se que, com base nessa vivência, os alunos aderiam melhor a disciplina, por conta da ABP (Cartagenes, et al., 2017; Meneses, et al., 2017).

Percebeu-se, também, o interesse e empenho dos alunos no desenvolvimento do projeto. Sendo para o docente foi uma forma interativa de administrar melhor o conteúdo e assim repassá-lo em sala de aula.

Podendo destacar como um ponto positivo da metodologia foi a adesão a disciplina na totalidade dos alunos durante as aulas, o que não ocorria na metodologia tradicional. Percebeu-se o interesse e empenho dos alunos no desenvolvimento do projeto. Sendo para o docente um ponto positivo na introdução da metodologia na referida disciplina.

Já a Pedagogia da metodologia tradicional é dirigir o educando para a sua formação intelectual e moral, tendo em vista, no futuro, assumir a sua posição individual na sociedade, de acordo com os ditames dessa sociedade (Silva, et al., 2014; Costa; Almeida \& Lopes, 2017; Cunha; Cunha \& Domingues, 2016). O que, com base na vivência desse estudo, por meio dessa metodologia, a evasão dos alunos às aulas de Biofísica era significativa.

Ademais, aliado as novas metodologias de aprendizagem, e a utilização das Tecnologias da Informação e Comunicação (TIC) foi fundamental como forma de complementar a formação das vídeoaulas (Delphino, et al., 2017; Marin, et al., 2010).

Como ponto negativo da experiência, foi necessário a utilização de recursos pessoais dos alunos, mesmo a instituição de ensino sendo privada não dispunha dos recursos, tornando uma limitação para o desenvolvimento da atividade proposta. Outro fator limitante, também, foi o manuseio dos recursos tecnológico por parte de alguns alunos, gerando uma certa restrição no desempenho do projeto. 
Outrossim, o conteúdo visual, diferentemente do escrito visto como mecanicista, motiva e aumenta a capacidade de retenção do que foi ensinado, exercitando as funções mentais e intelectuais do aluno. Outra verificação reside na motivação visual proporcionada pelo recurso referente às ilustrações atrativas, as geram interfaces visuais e auditivas com teor didáticopedagógicos favorecendo o desenvolvimento e construção da aprendizagem, sendo uma forma de aprendizado valorosa durante a vivência dos alunos (Oliveira, et al., 2017; Paiva, et al., 2016; Silva, et al., 2018).

Diante disso, as práticas pedagógicas atuais progressivamente rompem com os paradigmas tradicionais de ensino, as quais se baseavam na passividade do aluno diante da apresentação dos conteúdos que deveriam ser copiados, decorados e transcritos em uma avaliação, e vêm adotando ferramentas computacionais para a promoção da aprendizagem. O que tornou uma experiência com bom rendimento a Instituição do estudo.

Foi necessária, durante a vivência, adaptações na avaliação do módulo, visto que na metodologia tradicional os alunos ao término de cada módulo realizavam avaliações em formato de provas com questões objetivas e subjetivas. Já na ABP a avaliação foi por meio da construção dos projetos pela vídeoaula.

\section{Conclusão}

A compreensão acerca da percepção acadêmica sobre metodologias ativas através da ABP na disciplina de Biofísica, proporcionou a ampliação do conhecimento acadêmico por meio da vivência da criação de vídeoaulas no curso de Fisioterapia da IES em Belém-PA. Além de maximizar a adesão dos alunos às aulas de Biofísica, em comparação a metodologia tradicional. Além disso, o corpo docente também obteve uma melhor comunicação com os alunos, pois assim o conteúdo foi melhor explanado, reafirmando a sua importância na ABP.

Os novos métodos de aprendizagem têm sido um recurso adotado pelas instituições de ensino superior e, embora possa haver alguma limitação, os resultados parecem ser motivadores e favorecem a autonomia do educando. A metodologia ativa busca ofertar um ensino de qualidade com um caráter mais dinâmico e holístico, rompendo com o modo tradicional. O trabalho em equipe, a integração entre teoria e prática, desenvolvimento de uma visão crítica da realidade e promoção da autonomia e interdependência dos acadêmicos são os princípios desse novo método.

O conhecimento tanto das IES, quanto dos professores quando as metodologias ativas, sobretudo a ABP, ainda é ínfima, sendo necessário a efetivação dessas novas metodologias de ensino e, por consequência, melhorar a relação ensino-aprendizagem dos alunos. Sugere-se que novos trabalhos sobre a temática sejam desenvolvidos para maiores esclarecimentos à comunidade científica e acadêmica.

É necessário a construção de mais pesquisas que possam inserir outras disciplinas e, assim, obter uma análise mais multifatorial com um maior número de pessoas incluindo alunos e professores.

\section{Referências}

Alves, L. A., et al. (2021). Desafios e potencialidades da interprofissionalidade no contexto do programa de educação pelo trabalho para saúde. Research, Society and Development, 10(4), 1-9. http://dx.doi.org/10.33448/rsd-v10i4.14041.

Borges, T. S., \& Alencar, G. (2017). Metodologias Ativas na Promoção da Formação Crítica do Estudante: O Uso das Metodologias Ativas como Recurso Didático na Formação Crítica do Estudante do Ensino Superior. Cairu em Revista, 3(4), 119-143.

Brasil. Ministério da Saúde (2018). Programa de educação pelo trabalho para a saúde (PET-Saúde). https://antigo.saude.gov.br/images/pdf/2018/dezembro/1 3/Programa-de-Educacao-pelo-Trabalho-para-a-Saude-SaudeGraduaSUS-FINAL-WEB.pdf.

Cartagenes, M. V. et al. (2017). Software baseado no método ABA para auxílio ao ensino-aprendizagem de crianças portadoras de Transtorno Global do Desenvolvimento-Autista. Anais do Computer on the Beach, Itajaí, 162-171.

Costa, R. D. A., Almeida, C. M. M. \& Lopes, P. T. C. (2017). Aplicativos para dispositivos móveis e metodologias ativas: possibilidades pedagógicas para o ensino e aprendizagem em nível superior. Revista Educacional Interdisciplinar, Taquara, 6 (1), 1-9. 
Cunha, M. M., Cunha, S. N. \& Domingues, A. S. O. L. (2016). Contribuição dos textos, imagens, recursos audiovisuais, mapas conceituais e jogos eletrônicos no processo de explicação de conteúdos. Encontro Internacional de Formação de Professores e Fórum Permanente de Inovação Educacional, Aracaju, 9 (1), $1-14$.

Cecílio, L. C. O. \& Reis, A. A. O. (2018). Apontamentos sobre os desafios (ainda) atuais da atenção básica. Cadernos de saúde pública, 34 (8), 1-14. https://doi.org/10.1590/0102-311X00056917.

Delphino, F. B. et al. (2017). A utilização de metodologias ativas em cursos superiores para uma aprendizagem significativa. Innovando en Educación Superior: Experiencias clave en Latinoamérica y el Caribe, Santiago, 3 (1), 67-77.

Diesel, A., Baldez, A. L. S. \& Martins, S. N. (2017). Os princípios das metodologias ativas de ensino: uma abordagem teórica. Revista Thema, Pelotas (RS), 14 (1), 268-288.

Emery, L. R. \& Morgan, S. L. (2017). The application of project-based learning in bioinformatics training. PLoS Comput Biol., 13 (8).

Farias, D. N., Ribeiro, K. S. Q. S. \& Anjos, U. U. \& Brito, G. E. G. (2018). Interdisciplinaridade e interprofissionalidade na Estratégia Saúde da Família. Trab. Educ. Saúde, 16 (1), 141-162. http://dx.doi.org/10.1590/1981-7746-sol00098.

Grillo, L. P., Ibarra, L. S. \& Mezadri, T. (2019). Conceito ampliado de saúde na formação dos profissionais de educação física. Saúde e Pesquisa, 12 (2), 265273. https://doi.org/10.17765/2176-9206.2019v12n2p265-273.

Lima, V. V. (2017). Espiral Construtivista: Uma Metodologia Ativa de Ensino -Aprendizagem. Interface (Botucatu), Botucatu, 21 (61), 421-434.

Marin, M. J. S. et al. (2010). Aspectos das fortalezas e fragilidades no uso das metodologias ativas de aprendizagem. Rev. Bras. Educ. Méd., 34 (1), 13-20.

Meneses, T. Q., Faria, M. S., Silva, L. S., Cotta, R. M. M. \& Costa, G. D. (2017). A Utilização do Portfólio Reflexivo como Método de Ensino, Aprendizagem e Avaliação na Disciplina Políticas de Saúde. Journal of Management \& Primary Health Care, 7 (1), 149-149.

Moraes, J. P. \& Paim, C. F. (2017). O ensino da administração: práticas pedagógicas e seus impactos no desempenho profissional na visão dos formandos de graduação em administração de uma instituição privada na cidade de Porto Alegre - RS. Caminhos da Educação = Camine: WaysofEducation, , 9(2), 96-113.

Moraes, S. G., Marino, M. T. R. D., Fernandes, D. \& Façanha, M. C. (2017). Metodologias ativas: o protagonismo do discente no processo de aprendizagem. Os Desafios da Geografia Física na Fronteira do Conhecimento, 1, 3573-3578.

Moran, J. (2015). Mudando a educação com metodologias ativas. Coleção Mídias Contemporâneas. Convergências Midiáticas, Educação e Cidadania: aproximações jovens, 2, 15-33.

Oliveira, M. G. et al. (2017). Um curso de programação a distância com metodologias ativas e análise de aprendizagem por métricas de software. Revista Novas Tecnologias na Educação, 15(1), 1-10.

Paiva, M. R. F. et al. (2016). Metodologias ativas de ensino-aprendizagem: revisão integrativa. Revista de Políticas Públicas, 15(2), 145-153.

Ribeiro, J. B. P. et al. (2016). Intervenção pedagógica e metodologia ativa: o uso da instrução por colegas na educação profissional. Periódico Científico Outras Palavras, 2(12), 1-16

Rios, D. R. S., Sousa, D. A. B. \& Caputo, M. C. (2019). Diálogos interprofissionais e interdisciplinares na prática extensionista: o caminho para a inserção do conceito ampliado de saúde na formação acadêmica. Interface (Botucatu), 23. http://dx.doi.org/10.1590/interface.180080.

Schmitz, E. X. S., Reis, S. C. (2018). Sala de aula invertida: investigação sobre o grau de familiaridade conceitual teórico-prático dos docentes da universidade. Educação Temática Digital, 20(1), 153.

Silva, J. B. et al. (2018). Tecnologias digitais e metodologias ativas na escola: o contributo do Kahoot para gamificar a sala de aula. Revista Thema, 15(2), 780791.

Silva, L. S. et al. (2014). Formação de profissionais críticos-reflexivos: o potencial das metodologias ativas de ensino-aprendizagem e avaliação na aprendizagem significativa. Formação de profissionais críticos-reflexivos, metodologias ativas e aprendizagem significativa. Revista del Congrés Internacional de Docência Universitària i Innovación, Bellaterra, 2(1), 1-16.

Sobral, F. R. \& Campos, C. J. G. (2012). Utilização de Metodologia Ativa no Ensino e Assistência de Enfermagem na Produção Nacional: Revisão Integrativa. Rev. esc. enferm. 46(1), 208-218.

Vendruscolo, C., Trindade, L. L., Maffissoni, A. L., Martini, J. G., Silva Filho, C. C. \& Sandri, J. V. A. (2020). Implicação do processo de formação e educação permanente para atuação interprofissional. Rev Bras Enferm, 73 (2). https://dx.doi.org/10.1590/0034-7167-2018-0359. 